\title{
Distributed Finite-Time Optimal Resource Management for Microgrids Based on Multi-Agent Framework
}

DOI:

10.1109/TIE.2017.2721923

\section{Document Version}

Accepted author manuscript

Link to publication record in Manchester Research Explorer

\section{Citation for published version (APA):}

Zhao, T., \& Ding, Z. (2017). Distributed Finite-Time Optimal Resource Management for Microgrids Based on MultiAgent Framework. IEEE Transactions on Industrial Electronics, PP(99). https://doi.org/10.1109/TIE.2017.2721923

\section{Published in:}

IEEE Transactions on Industrial Electronics

\section{Citing this paper}

Please note that where the full-text provided on Manchester Research Explorer is the Author Accepted Manuscript or Proof version this may differ from the final Published version. If citing, it is advised that you check and use the publisher's definitive version.

\section{General rights}

Copyright and moral rights for the publications made accessible in the Research Explorer are retained by the authors and/or other copyright owners and it is a condition of accessing publications that users recognise and abide by the legal requirements associated with these rights.

\section{Takedown policy}

If you believe that this document breaches copyright please refer to the University of Manchester's Takedown Procedures [http://man.ac.uk/04Y6Bo] or contact uml.scholarlycommunications@manchester.ac.uk providing relevant details, so we can investigate your claim.

\section{OPEN ACCESS}




\title{
Distributed Finite-Time Optimal Resource Management for Microgrids Based on Multi-Agent Framework
}

\author{
Tianqiao Zhao, Zhengtao Ding, Senior Member, IEEE,
}

\begin{abstract}
This paper investigates the optimal resource management in a microgrid under various operating conditions. A two-level optimization system is proposed for the distributed optimal resource management based on a multi-agent system framework. The proposed strategy generates a reference of the optimal power output at the top-level through local communication. This strategy only requires the information among neighbouring participants without a central control coordination, and simultaneously accomplishes resource optimization in a finite-time while maintaining the supply-demand balance. The bottom-level control is responsible for the reference tracking of each corresponding participant in a microgrid. The convergent rate of the proposed algorithm is compared with other consensus-based algorithms through simulation studies. Simulation results in the IEEE 14-bus system and an actual islanded system are also presented to demonstrate the overall effectiveness of the proposed strategy.
\end{abstract}

Index Terms-Distributed optimization, consensus algorithm, finite-time stability, optimal resource management, multi-agent system, microgrid

\section{INTRODUCTION}

A microgrid is a cluster of various distributed generators (DGs), energy storage systems (ESSs), loads and control devices that can support a flexible and efficient electric network [1]. An intelligent microgrid is able to operate in both grid-connected mode and islanded mode [2]. Because of the intermittence of wind turbines (WTs) and photovoltaic power (PVs), a microgrid has to face new operational and control challenges of the resource management, especially in islanded operation mode, wherein it must maintain its own power supply-demand balance [3].

Due to the unpredictable nature of a microgrid, fast convergence algorithms are required to effectively coordinate both dispatchable and non-dispatchable energy resource to maintain microgrid stability [4], [5]. The task of the resource management can be defined to minimize the generation cost in a microgrid by coordinating the dispatch of energy resources, namely the conventional generation and energy storage to meet the demand economically. For this task, a proper algorithm is required to schedule power outputs of energy resources to meet

\footnotetext{
T. Zhao and Z. Ding are with the School of Electrica and Electronic Engineering, University of Manchester, Sackville Street Building, Manchester M13 9PL, UK (emails: tianqiao.zhao@postgrad.manchester.ac.uk; zhengtao.ding@manchester.ac.uk).
}

the load demand while maintaining system's constraints in a low-cost way.

Various research studies, such as lambda iteration method [6], the population dynamics approach [7], linear programming method [8] and particle swarm optimization [9], aimed at providing solutions to the resource management in demand response, economic generation dispatch, and loss minimization. All these methods require a powerful control centre to collect global information and process the amounts of received data [10]. In contrast, a distributed strategy only requires the information through a local communication network so that it can conduce to the development of the power system in a flexible and cost-effective way.

Recently, several distributed strategies have been presented to schedule power outputs of energy resources [11]-[17]. In [11] and [12], the authors presented a consensus+innovation framework and a consensus method for the economic dispatch problem in power systems, which employs the projection scheme to handle the inequality constraints. In [13], a consensus-based control strategy is proposed for scheduling the power output of distributed energy storage systems (ESSs). A leader agent is deployed to update power mismatch information, which makes the strategy not fully distributed. In [14], to minimize the economic cost, the authors proposed a distributed economic operation strategy to schedule various participants without considering the tripping of participants. As reported in [16], by modelling the DGs and loads as agents, the microgrid can be operated economically through the management of DGs and price-sensitive loads in a multi-agent system (MAS). Also, in [17], the active and reactive power of the islanded microgrid are dispatched optimally by a two-layer networked and distributed method in MAS framework.

In this paper, a novel distributed algorithm is proposed to solve the optimal resource management while respecting the generation constraints. In stead of using projection methods [18], [19] to deal with generation constraints, we apply a smooth $\epsilon$-exact penalty function [20] in problem formulation to handle the local constraints for the convenience of implementation. Additionally, the MAS framework is developed to be more compatible with the requirement of the power grid. By regarding each energy source as an agent, the agent in the proposed MAS framework only needs to exchange information with its neighbours through a directed communication network. As a result, the proposed strategy can work in a fully distributed way, which can reduce computational and 
communication burdens comparing with centralized methods. Furthermore, the proposed algorithm only requires a connected digraph so that the system can gain more robustness against the agent trip. It is worth noting that achieving a fast convergence is necessary for facilitating the development of a microgrid because the changes in a microgrid can be frequent and unpredicted [21]. A finite-time algorithm is adopted to achieve a higher convergence speed, which is beneficial to the lowinertial microgrid to maintain its power balance in the presence of unknown changes and agent trips. The effectiveness of the proposed distributed algorithm is validated through simulation studies in the IEEE-14 bus system and an actual islanded system.

The remainder part of the paper is organized as follows. Section II outlines the MAS system architecture and gives a description of the agent under a microgrid environment. Section III introduces a finite-time distributed optimal solution for an islanded microgrid. Section IV proposes a finite-time distributed optimal solution for a grid-connected microgrid. Section $\mathrm{V}$ presents the simulation results and corresponding analysis. Finally, the conclusion is drawn in Section VI.

\section{MULTI-AGENT SYSTEM ARCHITECTURE OF A MICROGRID}

In this section, the topology of the communication network for a microgrid is firstly introduced. Next, agents are defined in a microgrid context, and the cost function of each type of agents is formulated for distributed optimization.

\section{A. Multi-agent System Framework}

In our MAS framework, a microgrid consists of a utility grid, non-dispatchable DGs including WTs and PVs, conventional dispatchable synchronous generators (SGs), ESSs and non-controllable load demands. A Point of Common Coupling (PCC) of the utility grid is used to measure the power delivered/withdrawn and decide the operation mode of the microgrid. In the MAS framework, each participant in the microgrid is assigned to an intelligent agent that is able to interconnect with its adjacent agents to accomplish established objectives. A two-level control model, namely the top-level control and bottom-level control, is deployed to each agent as depicted in Fig. 1. The top-level control is a communication network for each agent to transfer information that is generated by an optimal strategy. Both the control mode and the physical agent platform are located at the bottom-level control. Following the control schemes in [15], the control mode is used to adjust the power output of the agent to the reference signal generated by the top-level, and the settings of different control modes can be decided by its operation condition locally. Meanwhile, the physical agent platform is the electrical components that to transmit the power generated/consumed.

The communication network $\mathcal{G}=(\mathcal{V}, \mathcal{E})$ over the microgrid is a weighted-balanced directed graph with $n$ agents, where $\mathcal{V}=\left\{\nu_{1}, \ldots, \nu_{n}\right\}$ denotes the agent set and $\mathcal{E}$ is the edge set. It is assumed that there exists a directed path that connects any pair of agents. The weight of an edge from $i$ th agent to $j$ th

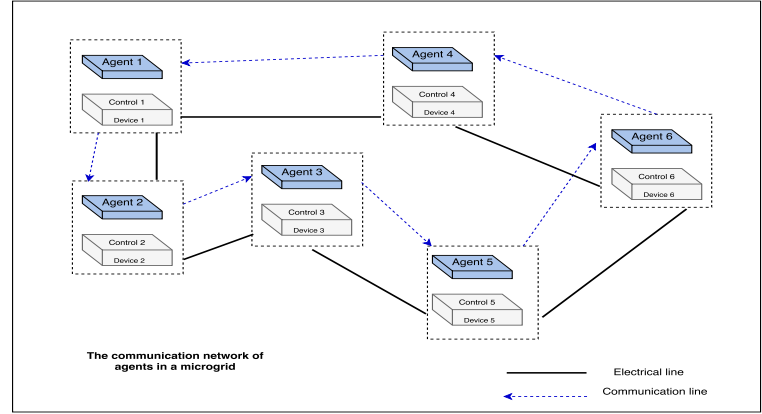

Fig. 1. The communication network of agents in a microgrid

agent is $a_{j i}>0$, and the weight on the self loop of $i$ th agent is $a_{i i}=0$. In the communication network, both dispatchable and non-dispatchable agents have ingoing and outgoing edges, and the weighted in-degree and out-degree of $i$ th agent are $d_{i n}(i)=\sum_{j=1}^{n} a_{i j}$ and $d_{\text {out }}(i)=\sum_{j=1}^{n} a_{j i}$, respectively. It should be noted that $d_{\text {out }}(\nu)=d_{\text {in }}(\nu)$ for all agents because the communication network is weighted-balanced. The Laplacian matrix $\mathcal{L}$ associates with $\mathcal{G}$ is defined as $\mathcal{L}_{i i}=\sum_{j \neq i} a_{i j}$ and $\mathcal{L}_{i j}=-a_{i j}, i \neq j$. It is worth noting that the designed communication network is independent of the power network [15]. A cost-effective communication network can be constructed based on the location, convenience of a practical microgrid.

\section{B. The Agent Description under MAS Framework}

In this section, agents are defined in the microgrid context under the proposed MAS framework, and the corresponding cost functions are defined for the algorithm design.

\section{Dispatchable Agents:}

Conventional Generator Agents: The conventional generator agents, includes fuel generators or gas plants. The generation cost of this type of agents is usually expressed as a typical quadratic function of active power output, i.e.,

$C_{k}\left(P_{G, k}\right)=\frac{1}{2} a_{k} P_{G, k}^{2}+b_{k} P_{G, k}+c_{k}, \quad P_{G, k}^{\min } \leq P_{G, k} \leq P_{G, k}^{\max }$

where non-negative $a_{k}, b_{k}$, and $c_{k}$ are the cost coefficients for $k$ th generator; $P_{G, k}^{\min }$ and $P_{G, k}^{\max }$ are the lower and upper bounds of the power output, respectively. The marginal cost function is the derivative of the cost function with respect to $P_{G, k}$, which is used to later algorithm design.

Storage Agents: The storage agents can be treated as a dispatchable agent since they have the capacity to transfer power to absorb excessive power and compensate insufficient power bidirectionally. Integrating storage agents to a microgrid may require additional costs, and thus, it is ideally to charge the storage agents when the marginal cost is low, and discharge them when the electricity rate is high otherwise [22]. Besides, battery life directly depends on different depth of discharging (DoD) scenarios in each cycle time [23], and the degradation cost is also an essential factor that may affect the economic decision for batteries [24]. To this end, inspired by [22], we 
proposed a general cost function for $j$ th battery by considering DoD:

$$
\begin{aligned}
& C_{j}\left(P_{B, j}\right)=\frac{1}{2} a_{j}\left(P_{B, j}+3 P_{B, j}^{\max }(\mathrm{DoD})\right)^{2} \\
& +b_{j}\left(\left(P_{B, j}+3 P_{B, j}^{\max }(\mathrm{DoD})\right)+c_{j}, \quad P_{B, j}^{\min } \leq P_{B, j} \leq P_{B, j}^{\max }\right.
\end{aligned}
$$

where $a_{j}, b_{j}$, and $c_{j}$ are the non-negative quadratic coefficients; $P_{B, j}^{\min }$ and $P_{B, j}^{\max }$ are the minimal and maximum charging rate, respectively. The DoD is the current depth of charge, and the marginal cost is defined as the derivative of the cost function with respect to $P_{B, j}$. From the cost function design, it is worth noting that the marginal cost is proportional to the DoD and the power withdrawn.

Utility Agent: When the microgrid is operated under the grid-connected mode, the utility agent will monitor the power transfer between the utility grid and the microgrid through the PCC. Accordingly, the microgrid updates its current electricity rate based on the broadcasting of the utility grid. The cost function can be assumed as a function with constant marginal cost [22],

$$
C_{U}=P_{r} \times P_{U}
$$

where $P_{r}$ is the current electricity rate and $P_{U}$ is the exchanged power between the utility and the microgrid. During the grid-connected mode operation, the marginal cost of each dispatchable agent should be equal to the marginal cost given by the utility grid, which equals to the electric rate.

\section{Nondispatchable Agents:}

Renewable Agents: The renewable generators (RGs) including WTs and PVs are considered as nondispachable since they are usually controlled in maximum peak power tracking (MPPT) mode. Therefore, the forecast of their generation cannot be accurate because of the stochastic and intermittency.

Load demand agents: The load demands, consisting of the industrial and residential loads, have the stochastic property of the consumer behaviours so that they cannot be treated as dispatchable due to the unprediction of power requirements.

Due to the intermittency of renewable generation, the operating condition of a microgrid may change frequently and unexpectedly. In order to ensure the performance and stability of the microgrid, the system should converge to the designed target in a timely manner. As a result, a faster convergence rate of a distributed optimal solution is required to meet the challenges of the microgrid development.

\section{FINITE-TIME DISTRIBUTED OPTIMAL SOLUTION FOR THE ISLANDED MICROGRID}

In this section, a finite-time distributed optimal solution is developed for the islanded microgrid to address the problems in the centralized strategies in the microgrid application.

The islanded microgrid should ensure the supply-demand balance in a cost-effective way. To this end, the objective for agents in an islanded microgrid is to balance the supply and demand, and minimize the generation/operation cost in the meantime. The objective function can be formulated as

$$
\begin{gathered}
\text { Min } \sum_{k \subseteq G} C_{k}\left(P_{G, k}\right)+\sum_{j \subseteq B} C_{j}\left(P_{B, j}\right) \\
\text { s.t } \quad \\
P_{D}=P_{L}-P_{R}=\sum_{k \subseteq G} P_{G, k}+\sum_{j \subseteq B} P_{B, j} \\
P_{G, k}^{\min } \leq P_{G, k} \leq P_{G, k}^{\max } \\
P_{B, j}^{\min } \leq P_{B, j} \leq P_{B, j}^{\max }
\end{gathered}
$$

where $P_{D}$ is the net power demand; $P_{L}$ and $P_{R}$ are all demands of load agents and all nondispatchable generations, respectively.

For convenience, we denote $P_{i}$ as the output power, and $P_{i}^{\min }$ and $P_{i}^{\max }$ as the lower and upper bound of $i$ th dispatchable agent, respectively. We further define $\omega_{i}, \sigma_{i}$ and $c_{i}$ as the coefficients of $i$ th cost function. Then (4) can be written as

$$
\begin{array}{lc}
\text { Min } & \sum_{i \in n} C_{i}\left(P_{i}\right) \\
\text { s.t } & \sum_{i \in n} P_{i}=P_{L}-P_{R} \\
& h_{i}\left(P_{i}\right)=\left(P_{i}^{\text {min }}-P_{i}\right)\left(P_{i}^{\text {max }}-P_{i}\right) \leq 0 .
\end{array}
$$

The solution for the optimal resource management can be obtained by a centralized approach, which requires a control centre and bi-directional communication lines between the controller and the connected agents. The central controller collects all data firstly, e.g., the cost functions, the local constraints, and the power generated/consumed. Then, it solves the optimal problem and broadcasts the solution to all agents. However, due to the high penetration of RGs, more frequent updates and a higher convergence speed are required. The centralized strategy may lose its control efficiency if operating conditions change frequently and unpredictably. To address this problem, the following sections will introduce two distributed solutions for the optimal resource management.

\section{A. Alternative Formulation}

Inequality constraints may cause difficulties in the optimal control design. Before presenting the optimal solution, an alternative formulation of the optimization problem is developed for dealing with inequality constraints by using $\epsilon$-exact penalty function proposed in [20], i.e.,

$$
p_{\epsilon, i}\left(h_{i}\left(P_{i}\right)\right)=\left\{\begin{array}{lc}
0, & h_{i}\left(P_{i}\right) \leq 0 \\
\frac{h_{i}\left(P_{i}\right)^{2}}{2 \epsilon}, & 0 \leq h_{i}\left(P_{i}\right) \leq \epsilon \\
h_{i}\left(P_{i}\right)-\frac{\epsilon}{2}, & h_{i}\left(P_{i}\right) \geq \epsilon
\end{array}\right.
$$

where $\epsilon$ is a positive coefficient. Thus the objective function is further written as

$$
\begin{aligned}
\text { Min } & C_{\epsilon}(P) & =\sum_{i \in n} C_{\epsilon, i}\left(P_{i}\right) \\
\text { s.t } & \sum_{i \in n} P_{i} & =P_{L}-P_{R}
\end{aligned}
$$


where $C_{\epsilon, i}\left(P_{i}\right)=C_{i}\left(P_{i}\right)+\mu p_{\epsilon, i}\left(h_{i}\left(P_{i}\right)\right)$, and $P=$ $\left[P_{1}, \ldots, P_{n}\right]^{T} \cdot \mu$ is a positive weight of the penalty function.

Let $P^{*}=\left[P_{1}^{*}, \ldots, P_{n}^{*}\right]^{T}$ be the optimal solution for (5), and $\hat{P}^{*}=\left[\hat{P}_{1}^{*}, \ldots, \hat{P}_{n}^{*}\right]^{T}$ be the solution for (6). Then, following Proposition 6 in [20], for $\mu=\frac{1-n}{1-\sqrt{n}} \mu^{*}$, the relationship between (5) and (6) is

$$
0 \leq C\left(P^{*}\right)-C_{\epsilon}\left(\hat{P}^{*}\right) \leq \epsilon \mu n,
$$

where $\mu^{*}>\max \left\{\eta^{*}\right\}$ with $\eta^{*}=\left\{\eta_{1}^{*}, \ldots, \eta_{n}^{*}\right\}$ is the Lagrange multiplier vector satisfying the KKT condition [25]. According to the Proposition 4 in [26], the upper bound of $\eta^{*}$ is given as

$$
\max \left\{\eta_{i}^{*}\right\}_{i=1}^{n} \leq \frac{2 \max \left\{\max _{P_{i} \in P_{\text {fea }, i}}\left|\nabla C_{i}\left(P_{i}\right)\right|\right\}_{i=1}^{n}}{\min \left\{P_{i}^{M}-P_{i}^{m}\right\}_{i=1}^{n}},
$$

where $\nabla C_{i}\left(P_{i}\right)$ is the gradient of $C_{i}\left(P_{i}\right)$, and $P_{\text {fea }, i}=\left\{P_{i} \in\right.$ $\mathcal{R} \mid \sum_{i \in n} P_{i}=P_{L}-P_{R}$ and $\left.\left(P_{i}^{\min }-P_{i}\right)\left(P_{i}^{\max }-P_{i}\right) \leq 0\right\}$, for $i \in n$.

Remark 3.1: To ensure the $\epsilon$-solution is equal to the solution of the original optimization problem exactly, the local bound of each dispatchable agent is further modified as $h_{i}\left(P_{i}\right)=$ $\left(P_{i}^{\min }-P_{i}\right)\left(P_{i}^{\max }-P_{i}\right)+\epsilon \leq 0$ that can meet the accurate requirement of the system. Note that $\epsilon$ can be chosen to be significantly small that does not affect the convergence of the original problem. After the change of local bounds, the $\epsilon$ feasible set is the same as the feasible set of the original optimization problem. The proof can be obtained following a similar manner from [20].

\section{B. Finite-time distributed optimal energy management}

By invoking the $\epsilon$-penalty function, a finite-time distributed optimal solution is proposed to solve the optimization problem (6). The update dynamics for $i$ th dispatchable agent is

$$
\begin{aligned}
\dot{P}_{i}= & \sum_{j \in n} a_{i j}\left(\nabla C_{\epsilon, j}\left(P_{j}\right)-\nabla C_{\epsilon, i}\left(P_{i}\right)\right)^{2-\frac{p}{q}} \\
& +\sum_{j \in n} a_{i j}\left(\nabla C_{\epsilon, j}\left(P_{j}\right)-\nabla C_{\epsilon, i}\left(P_{i}\right)\right)^{\frac{p}{q}}
\end{aligned}
$$

where $p$ and $q$ are the positive odd integers satisfying $p<q$. The dynamics is distributed in the sense that each dispatchable agent only requires local available information and the information from its adjacent agents through a local communication network.

Theorem 3.1: Consider the optimization problem in (6) with the dynamics (9), the feasible set $P_{\text {fea }}$ is time-invariant, and any trajectory starting from $P_{\text {fea }}$ converges to the solution set of (6) in a finite-time.

Proof: A candidate function $V_{1}=\left|\sum_{i=1}^{n} P_{i}\right|$ is considered since the active power of ESSs would be negative/positive when they are charged/discharged. Also it should be noted that $P_{D} \neq 0$, we only consider the cases for $P_{D}>0$ or $P_{D}<0$ accordingly. Firstly, if $\sum_{i=1}^{n} P_{i}>0, V_{1}=\sum_{i=1}^{n} P_{i}$ and the derivative of $V_{1}$ is

$$
\begin{aligned}
\dot{V}_{1} & =\sum_{i=1}^{n} \sum_{j \in n} a_{i j}\left(\nabla C_{\epsilon, j}\left(P_{j}\right)-\nabla C_{\epsilon, i}\left(P_{i}\right)\right)^{2-\frac{p}{q}} \\
& +\sum_{i=1}^{n} \sum_{j \in n} a_{i j}\left(\nabla C_{\epsilon, j}\left(P_{j}\right)-\nabla C_{\epsilon, i}\left(P_{i}\right)\right)^{\frac{p}{q}}=0 .
\end{aligned}
$$

Then, if $\sum_{i=1}^{n} P_{i}<0, V_{1}=-\sum_{i=1}^{n} P_{i}$ and the derivative is

$$
\begin{aligned}
\dot{V}_{1} & =-\left(\sum_{i=1}^{n} \sum_{j \in n} a_{i j}\left(\nabla C_{\epsilon, j}\left(P_{j}\right)-\nabla C_{\epsilon, i}\left(P_{i}\right)\right)^{2-\frac{p}{q}}\right. \\
& \left.+\sum_{i=1}^{n} \sum_{j \in n} a_{i j}\left(\nabla C_{\epsilon, j}\left(P_{j}\right)-\nabla C_{\epsilon, i}\left(P_{i}\right)\right)^{\frac{p}{q}}\right)=0 .
\end{aligned}
$$

From the above results, it concludes that the total power output of network is conserved and the feasible set $P_{\text {fea }}$ is timeinvariant.

Next, we show that the trajectories starting from $P_{\text {fea }}$ will converge to the solution set in a finite-time manner. Note that the solution to (6) is unique so that $P^{*}$ will be the unique solution to the minimization problem. Denote $V_{2}=$ $\sum_{i=1}^{n} C_{\epsilon, i}\left(P_{i}\right)-\sum_{i=1}^{n} C_{\epsilon, i}\left(P_{i}^{*}\right)$ as a candidate function. It is worth noting that $V_{2} \geq 0$, and $V_{2}=0$ when $P_{i}=P^{*}$. From (9), and let $\xi_{i} \in \nabla C_{\epsilon, i}\left(P_{i}\right)$,

$$
\begin{aligned}
\dot{V}_{2}= & \sum_{i=1}^{n} \xi_{i} \dot{P}_{i} \\
= & \sum_{i=1}^{n} \xi_{i}\left[\sum_{j \in n} a_{i j}\left(\xi_{j}-\xi_{i}\right)^{2-\frac{p}{q}}+\sum_{j \in n} a_{i j}\left(\xi_{j}-\xi_{i}\right)^{\frac{p}{q}}\right] \\
= & \frac{1}{2} \sum_{i, j=1}^{n} a_{i j}\left(\xi_{i}-\xi_{j}\right)\left(\xi_{j}-\xi_{i}\right)^{2-\frac{p}{q}} \\
& +\frac{1}{2} \sum_{i, j=1}^{n} a_{i j}\left(\xi_{i}-\xi_{j}\right)\left(\xi_{j}-\xi_{i}\right)^{\frac{p}{q}} \\
= & -\frac{1}{2} \sum_{i, j=1}^{n} a_{i j}\left(\xi_{j}-\xi_{i}\right)^{\frac{3 q-p}{q}} \\
& -\frac{1}{2} \sum_{i, j=1}^{n} a_{i j}\left(\xi_{j}-\xi_{i}\right)^{\frac{q+p}{q}} \\
= & -\frac{1}{2} \sum_{i, j=1}^{n}\left[a_{i j}^{\frac{2 q}{3 q-p}}\left(\xi_{j}-\xi_{i}\right)^{2}\right]^{\frac{3 q-p}{2 q}} \\
& -\frac{1}{2} \sum_{i, j=1}^{n}\left[a_{i j}^{\frac{2 q}{q+p}}\left(\xi_{j}-\xi_{i}\right)^{2}\right]^{\frac{q+p}{2 q}}
\end{aligned}
$$

Following the Corollary 2 in [27] and Lemma 3.4 in [28], it can be obtained that

$$
\begin{aligned}
\dot{V}_{2} & \leq-\frac{1}{2} n^{\frac{p-q}{q}} \sum_{i, j=1}^{n}\left[a_{i j}^{\frac{2 q}{3 q-p}}\left(\xi_{j}-\xi_{i}\right)^{2}\right]^{\frac{3 q-p}{2 q}} \\
& -\frac{1}{2}\left[\sum_{i, j=1}^{n} a_{i j}^{\frac{2 q}{q+p}}\left(\xi_{j}-\xi_{i}\right)^{2}\right]^{\frac{q+p}{2 q}} \\
& =-\frac{1}{2} n^{\frac{p-q}{q}}\left[2 \xi^{T} \mathcal{L}_{B} \xi\right]^{\frac{3 q-p}{2 q}}-\frac{1}{2}\left[2 \xi^{T} \mathcal{L}_{C} \xi\right]^{\frac{q+p}{2 q}}
\end{aligned}
$$

where $\xi=\left[\xi_{1}, \ldots, \xi_{n}\right]^{T}$. Consider the weighted-balance directed graphs $\mathcal{G}_{B}$ and $\mathcal{G}_{C}$ with the adjacency matrices $\mathcal{A}_{B}=\left[a_{i j}^{B}\right]_{n \times n}$ and $\mathcal{A}_{C}=\left[a_{i j}^{C}\right]_{n \times n}$, respectively. Then $\mathcal{L}_{B}$ 
and $\mathcal{L}_{C}$ are the corresponding Laplacian matrices of $\mathcal{G}_{B}$ and $\mathcal{G}_{C}$ respectively. Define

$$
\begin{aligned}
& \Gamma_{B}=2 \xi^{T} \mathcal{L}_{B} \xi \geq 2 \lambda_{2}\left(\mathcal{L}_{B}\right)\left\|\xi-\frac{1}{n}\left(\mathbf{1}_{n}^{T} \xi\right) \mathbf{1}_{n}^{T}\right\|_{2}^{2} \\
& \Gamma_{C}=2 \xi^{T} \mathcal{L}_{C} \xi \geq 2 \lambda_{2}\left(\mathcal{L}_{C}\right)\left\|\xi-\frac{1}{n}\left(\mathbf{1}_{n}^{T} \xi\right) \mathbf{1}_{n}^{T}\right\|_{2}^{2} .
\end{aligned}
$$

Since $C_{\epsilon}(P)$ is strongly convex, for $\hat{P}=\left[\hat{P}_{1}, \ldots, \hat{P}_{n}\right]$, it follows that

$C_{\epsilon}(\hat{P}) \geq C_{\epsilon}(P)+\left(\xi-\frac{1}{n}\left(\mathbf{1}_{n}^{T} \xi\right) \mathbf{1}_{n}^{T}\right)(\hat{P}-P)+\frac{k}{2}\|\hat{P}-P\|^{2}$.

It is worth noting that the minimum of (16) is $C_{\epsilon}(P)-$ $\frac{1}{2 k}\left\|\xi-\frac{1}{n}\left(\mathbf{1}_{n}^{T} \xi\right) \mathbf{1}_{n}^{T}\right\|$ for constant $P$. For $\hat{P}=P^{*}$, we have $V_{2} \leq-\frac{1}{2 k}\left\|\xi-\frac{1}{n}\left(\mathbf{1}_{n}^{T} \xi\right) \mathbf{1}_{n}^{T}\right\|$. Therefore,

$$
\begin{aligned}
\dot{V}_{2} & \leq-\frac{1}{2} n^{\frac{p-q}{q}}\left[4 k \lambda_{2}\left(\mathcal{L}_{B}\right) V_{2}\right]^{\frac{3 q-p}{2 q}}-\frac{1}{2}\left[4 k \lambda_{2}\left(\mathcal{L}_{C}\right) V_{2}\right]^{\frac{q+p}{2 q}} \\
& \leq-\frac{1}{2} n^{\frac{p-q}{q}}\left[4 k \bar{\lambda} V_{2}\right]^{\frac{3 q-p}{2 q}}-\frac{1}{2}\left[4 k \bar{\lambda} V_{2}\right]^{\frac{q+p}{2 q}} \\
& =-\frac{1}{2}\left[n^{\frac{p-q}{q}}\left[4 k \bar{\lambda} V_{2}\right]^{\frac{q-p}{q}}+1\right]\left(\left[4 k \bar{\lambda} V_{2}\right]^{\frac{q+p}{2 q}}\right)
\end{aligned}
$$

where $\bar{\lambda}=\min \left\{\lambda_{2}\left(\mathcal{L}_{B}\right), \lambda_{2}\left(\mathcal{L}_{C}\right)\right\}>0$. Let $\delta=\sqrt{4 k \bar{\lambda} V_{2}}$, then

$$
\dot{\delta}=-n^{\frac{p-q}{q}} \bar{\lambda} \delta^{\frac{2 q-p}{q}}-\bar{\lambda} \delta^{\frac{p}{q}} .
$$

With Lemma 4.1 in [28] and Comparison Lemma in [29], we conclude that

$$
\lim _{t \rightarrow T_{1}} V_{2}=0,
$$

where $T_{1}$ is given by

$$
T_{1} \leq \frac{\pi q n^{\frac{q-p}{2 q}}}{2 \bar{\lambda}(q-p)} .
$$

Note that $V_{2} \rightarrow 0$ implies $\sum_{i=1}^{n} C_{\epsilon, i}\left(P_{i}\right)-\sum_{i=1}^{n} C_{\epsilon, i}\left(P_{i}^{*}\right)=$ 0 , which can deduce that $\xi \in \operatorname{span}\left\{\mathbf{1}_{n}\right\}$. Since $\mathbf{1}_{n}^{T} P_{0}=$ $P_{L}-P_{R}$ with $P_{0}=\left[P_{1,0}, \ldots, P_{n, 0}\right]^{T}$, it concludes that the trajectories starting from $P_{\text {fea }}$ converge to the set of solutions of the optimal problem.

\section{FINITE-TIME DISTRIBUTED SOLUTION FOR GRID-CONNECTED MICROGRID}

Once the operation mode of microgrid is switched to the grid-connected mode, the participants in the microgrid should follow the marginal cost set by the utility agent, which can be treated as a tracking problem, i.e.,

$$
\nabla C_{\epsilon, 1}\left(P_{1}\right)=\nabla C_{\epsilon, 2}\left(P_{2}\right)=\cdots=\nabla C_{\epsilon, n}\left(P_{n}\right)=\nabla C_{U} .
$$

To address this problem, a finite-time distributed solution is proposed as

$$
\begin{aligned}
\dot{P}_{i}= & \sum_{j \in n} a_{i j}\left(\nabla C_{\epsilon, j}\left(P_{j}\right)-\nabla C_{\epsilon, i}\left(P_{i}\right)\right)^{2-\frac{p}{q}} \\
& +\sum_{j \in n} a_{i j}\left(\nabla C_{\epsilon, j}\left(P_{j}\right)-\nabla C_{\epsilon, i}\left(P_{i}\right)\right)^{\frac{p}{q}} \\
& +g_{i}\left(P_{r}-\nabla C_{\epsilon, i}\left(P_{i}\right)\right)^{2-\frac{p}{q}}+g_{i}\left(P_{r}-\nabla C_{\epsilon, i}\left(P_{i}\right)\right)^{\frac{p}{q}}
\end{aligned}
$$

where $P_{r}=\nabla C_{U}$, and $g_{i}$ is the pinning gain of $i$ th dispatchable agent. Define $e_{i}=P_{r}-\xi_{i}$, where $\xi_{i} \in \nabla C_{\epsilon, i}\left(P_{i}\right)$, and then the derivative of $e_{i}$ is given as

$$
\begin{aligned}
\dot{e}_{i}=\dot{\xi}_{i} \cdot \dot{P}_{i} & =\omega_{i}\left[\left(\sum_{j \in n} a_{i j}\left(\xi_{j}-\xi_{i}\right)^{2-\frac{p}{q}}+\sum_{j \in n} a_{i j}\left(\xi_{j}-\xi_{i}\right)^{\frac{p}{q}}\right)\right. \\
& \left.\left.+g_{i}\left(P_{r}-\xi_{i}^{2-\frac{p}{q}}\right)+g_{i}\left(P_{r}-\xi_{i}\right)^{\frac{p}{q}}\right)\right] \\
& =\omega_{i}\left[\left(\sum_{j \in n} a_{i j}\left(e_{j}-e_{i}\right)^{2-\frac{p}{q}}+\sum_{j \in n} a_{i j}\left(e_{j}-e_{i}\right)^{\frac{p}{q}}\right)\right. \\
& \left.-g_{i}\left(e_{i}\right)^{2-\frac{p}{q}}-g_{i}\left(e_{i}\right)^{\frac{p}{q}}\right]
\end{aligned}
$$

where $\nabla^{2} C_{i}\left(P_{i}\right)=\omega_{i}>0$. Suppose $\omega_{i}$ is known by $i$ th dispatchable agent, and the communication network satisfies the condition in weights that $\omega_{i} a_{i j}=\omega_{j} a_{j i}$.

Theorem 4.1: Suppose the weighted-balance direct graph is connected. Then, by applying (22), the distributed tracking problem (21) is solved in a finite-time.

Proof: Consider a candidate Lyapunov function, $V_{3}=$ $\frac{1}{2} \sum_{i=1}^{n} e_{i} \cdot e_{i}$, and then differentiating $V_{3}$ yields

$$
\begin{aligned}
\dot{V}_{3}= & \sum_{i=1}^{n} e_{i} \omega_{i}\left[\left(\sum_{j \in n} a_{i j}\left(e_{j}-e_{i}\right)^{2-\frac{p}{q}}+\sum_{j \in n} a_{i j}\left(e_{j}-e_{i}\right)^{\frac{p}{q}}\right)\right. \\
& \left.-g_{i}\left(e_{i}\right)^{2-\frac{p}{q}}-g_{i}\left(e_{i}\right)^{\frac{p}{q}}\right] \\
= & \frac{1}{2} \sum_{i, j=1}^{n}\left[\left(\omega_{i} a_{i j}\right)\left(e_{i}-e_{j}\right)\left(e_{j}-e_{i}\right)^{2-\frac{p}{q}}\right]- \\
& +\frac{1}{2} \sum_{i, j=1}^{n}\left[\left(\omega_{i} a_{i j}\right)\left(e_{i}-e_{j}\right)\left(e_{j}-e_{i}\right)^{\frac{p}{q}}\right. \\
& -\sum_{i=1}^{n}\left[\left(\omega_{i} g_{i}\right)\left(e_{i}\right)^{\frac{3 q-p}{q}}-\sum_{i=1}^{n}\left[\left(\omega_{i} g_{i}\right)\left(e_{i}\right)^{\frac{q+p}{q}}\right.\right. \\
= & -\frac{1}{2} \sum_{i, j=1}^{n}\left[\left(\omega_{i} a_{i j}\right)^{\frac{2 q}{3 q-p}}\left(e_{j}-e_{i}\right)^{2}\right]^{\frac{3 q-p}{2 q}} \\
& -\frac{1}{2} \sum_{i, j=1}^{n}\left[\left(\omega_{i} a_{i j}\right)^{\frac{2 q}{q+p}}\left(e_{j}-e_{i}\right)^{2}\right]^{\frac{q+p}{2 q}} \\
& -\sum_{i=1}^{n}\left[\left(\omega_{i} g_{i}\right)^{\frac{2 q}{3 q-p}}\left(e_{i}\right)^{2}\right]^{\frac{3 q-p}{2 q}}-\sum_{i=1}^{n}\left[\left(\omega_{i} g_{i}\right)^{\frac{2 q}{q+p}}\left(e_{i}\right)^{2}\right]^{\frac{q+p}{2 q}} .
\end{aligned}
$$

As a result,

$$
\begin{aligned}
\dot{V}_{3} \leq & -\frac{1}{2}\left(\sum_{i, j=1}^{n}\left(\omega_{i} a_{i j}\right)^{\frac{2 q}{3 q-p}}\left(e_{j}-e_{i}\right)^{2}+2 \sum_{i=1}^{n}\left(\omega_{i} g_{i}\right)^{\frac{2 q}{3 q-p}}\left(e_{i}\right)^{2}\right)^{\frac{3 q-p}{2 q}} \\
& -\frac{1}{2}\left(\sum_{i, j=1}^{n}\left(\omega_{i} a_{i j}\right)^{\frac{2 q}{q+p}}\left(e_{j}-e_{i}\right)^{2}+2 \sum_{i=1}^{n}\left(\omega_{i} g_{i}\right)^{\frac{2 q}{q+p}}\left(e_{i}\right)^{2}\right)^{\frac{q+p}{2 q}} .
\end{aligned}
$$


Define

$$
\begin{aligned}
& \Theta=\sum_{i, j=1}^{n}\left(\omega_{i} a_{i j}\right)^{\frac{2 q}{3 q-p}}\left(e_{j}-e_{i}\right)^{2}+2 \sum_{i=1}^{n}\left(\omega_{i} g_{i}\right)^{\frac{2 q}{3 q-p}}\left(e_{i}\right)^{2} \\
& \Phi=\sum_{i, j=1}^{n}\left(\omega_{i} a_{i j}\right)^{\frac{2 q}{q+p}}\left(e_{j}-e_{i}\right)^{2}+2 \sum_{i=1}^{n}\left(\omega_{i} g_{i}\right)^{\frac{2 q}{q+p}}\left(e_{i}\right)^{2} .
\end{aligned}
$$

Let $\mathcal{G}_{\alpha}$ and $\mathcal{G}_{\beta}$ be the weighted-balance direct graphs with $\alpha_{i j}=\left(\omega_{i} a_{i j}\right)^{\frac{2 q}{3 q-p}}$ and $\beta_{i j}=\left(\omega_{i} a_{i j}\right)^{\frac{2 q}{q+p}}$, respectively. The Laplacian matrix of each graph is $\mathcal{L}_{\alpha}$ and $\mathcal{L}_{\beta} . G_{b}=\operatorname{diag}\left(g_{b, i}\right)$ with $g_{b, i}=\left(\omega_{i} g_{i}\right)^{\frac{2 q}{3 q-p}}$, and $G_{c}=\operatorname{diag}\left(g_{c, i}\right)$ with $g_{b, i}=$ $\left(\omega_{i} g_{i}\right)^{\frac{2 q}{q+p}}$ are the diagonal matrix of pinning gains of each graph. Then, following Lemma 4 in [30], one has

$$
\begin{aligned}
& \Theta=2 e\left(\mathcal{L}_{\alpha}+G_{b}\right) e \geq 2 \lambda_{1}\left(\mathcal{L}_{\alpha}+G_{b}\right) e^{T} e>0 \\
& \Phi=2 e\left(\mathcal{L}_{\beta}+G_{c}\right) e \geq 2 \lambda_{1}\left(\mathcal{L}_{\beta}+G_{c}\right) e^{T} e>0
\end{aligned}
$$

which can be rewritten as $4 \lambda_{1}\left(\mathcal{L}_{\alpha}+G_{b}\right) \leq \frac{\Theta}{V_{3}}$ and $4 \lambda_{1}\left(\mathcal{L}_{\beta}+\right.$ $\left.G_{c}\right) \leq \frac{\Phi}{V_{3}}$. Then, following the similar lines in the proof of Theorem 3.1, (24) can be reformulated as

$$
\begin{aligned}
\dot{V}_{3} & \leq-\frac{1}{2} n^{\frac{p-q}{q}}\left[4 \lambda_{1}\left(\mathcal{L}_{\alpha}+G_{b}\right)\right]^{\frac{3 q-p}{2 q}}-\frac{1}{2}\left[4 \lambda_{1}\left(\mathcal{L}_{\beta}+G_{c}\right)\right]^{\frac{q+p}{2 q}} \\
& \leq-\frac{1}{2} n^{\frac{p-q}{q}}\left[4 \overline{\lambda_{1}}\right]^{\frac{3 q-p}{2 q}}-\frac{1}{2}\left[4 \overline{\lambda_{1}}\right]^{\frac{q+p}{2 q}}
\end{aligned}
$$

where $\overline{\lambda_{1}}=\min \left\{\lambda_{1}\left(\mathcal{L}_{\alpha}+G_{b}\right), \lambda_{1}\left(\mathcal{L}_{\beta}+G_{c}\right)\right\}$. Similarly, with Lemma 4.1 in [28] and Comparison Lemma in [29], there is

$$
\lim _{t \rightarrow T_{2}} V_{3}=0,
$$

where $T_{2}$ is given by

$$
T_{2} \leq \frac{\pi q n^{\frac{q-p}{2 q}}}{2 \overline{\lambda_{1}}(q-p)} .
$$

Note that $V_{3} \rightarrow 0$ implies $e_{i} \rightarrow 0$, i.e., $\nabla C_{\epsilon, i}\left(P_{i}\right) \rightarrow P_{r}$. Thus the tracking problem (21) is solved in a finite-time.

\section{A. Algorithm Implementation}

As previous mentioned, the proposed algorithm can be implemented in a MAS framework. Each agent in the proposed MAS framework consists of a two-level control model as shown in Fig. 1. Each agent communicates with its neighbours by the top-level control to discover the solution of the optimal resource management and generate a reference output for bottom-level control. As shown in [15], the communication network can be designed independently in a cost-effective approach based on the location, convenience of a real application.

Once the power reference is generated, the bottom-level control is implemented to control the agent to track this reference, which can follow the control scheme introduced in [31].

Remark 4.1: Note that the upper bounds of the settlingtime $T_{1}$ and $T_{2}$ only depend on the designed parameters $p, q$, the communication network structure and the volume $n$ of the given system. As a result, the convergence time can be estimated based on the algorithm and network design.

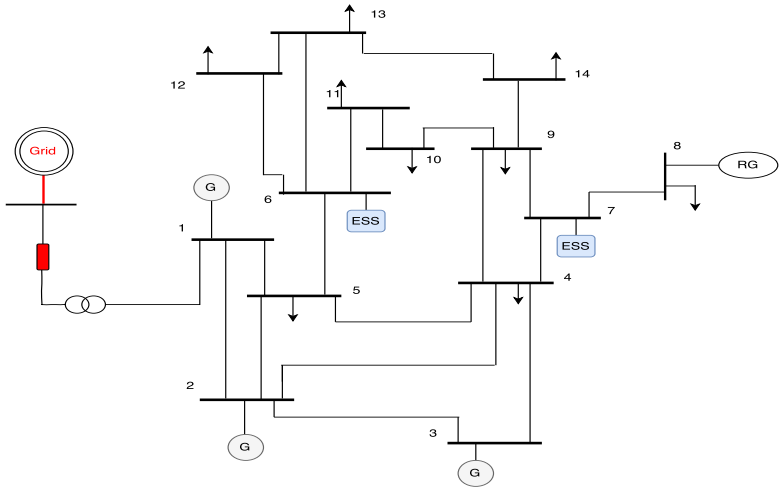

Fig. 2. IEEE 14-bus system

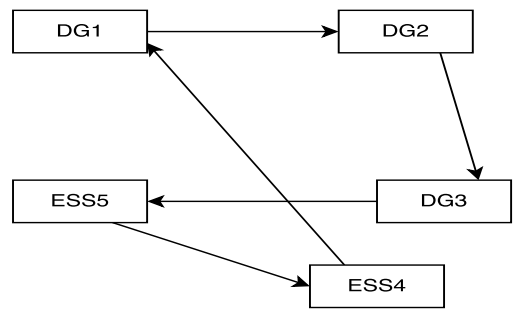

Fig. 3. Communication diagram

\section{Simulation Results AND ANALYSis}

In this section, three case studies are provided to verify the effectiveness of the proposed finite-time algorithm. The simulation studies are carried out in IEEE 14-bus system, where Fig. 2 depicts the topology of the test system that includes three conventional generators, two storage systems, one renewable generator and nine loads. Table. I gives the parameters of each type of agents, and Fig. 3 indicates the communication diagram of the dispatchable agents.

In Case 5.1, to verify the faster convergence property, the proposed finite-time algorithm is compared with other two optimization solutions for the resource management in microgrids. Case 5.2 investigates the performance of the proposed strategy under difference conditions of the microgrid operation. In case 5.3, an actual islanded system is built in MATLAB/Simulink to test the proposed algorithm. Finally, a modified IEEE 162-bus system [32] is adopted for verifying the scalability of the proposed algorithm.

\section{A. Case 5.1}

The resource management has been solved in many works by using the MAS framework. In [33], the authors proposed a

TABLE I

PARAMETERS OF SIMULATION STUDIES

\begin{tabular}{lllll}
\hline & $\omega_{i}$ & $\sigma_{i}$ & $P_{i}^{\min }$ & $P_{i}^{\max }$ \\
\hline G1 & 0.082 & 3.25 & 20 & 65 \\
G2 & 0.068 & 4.2 & 20 & 65 \\
G3 & 0.071 & 5.08 & 20 & 70 \\
ESS1 & 0.3 & 0.07 & -45 & 45 \\
ESS2 & 0.4 & 0.062 & -50 & 50 \\
\hline
\end{tabular}




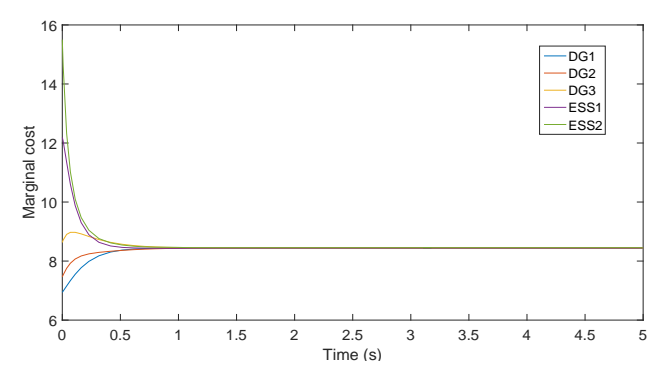

Fig. 4. The marginal cost update with the proposed finite-time algorithm

distributed gradient algorithm (DGA) for the maximize social welfare in a microgrid by considering the actual situation of renewable energy. Also, a Laplacian-gradient dynamics (LGD) in [34] is proposed for the economic dispatch problem, which achieves the supply-demand balance while minimizing the total generation cost. However, [33] solves the problem in the undirected graph and the fast convergence requirement of a microgrid is not considered in [34]. In contrast, this paper solves the optimal resource management in a finitetime approach. To demonstrate the fast convergence property of the proposed algorithm, the proposed finite-time algorithm is compared with the DGA and the LGD in this case firstly. The communication network keeps the same configuration and is supposed to be connected during the simulation study. Fig. 4 shows the performance of the proposed finite-time algorithm. The results indicate that both marginal cost of each dispatchable agent converges to the optimal value in a finite-time. To give a marked comparison, Fig. 5 and 6 depict the comparative evaluation of the marginal cost update. Furthermore, as shown in Table. II, the setting time of the proposed algorithm (1.2s) is shorter compared with the DGA (4.3s) and LGD (5.9s). As the results shown, the proposed algorithm leads to a faster convergence compared with the algorithm proposed in [33] and [34], which is an indispensable part for the microgrid operation because of the intermittent and unpredictable of non-dispatchable agents.

TABLE ॥

THE SETTING TIME OF DIFFERENT ALGORITHMS

\begin{tabular}{cccc}
\hline & Finite-time & DGA & LGD \\
\hline $\mathrm{T}$ & $1.2 \mathrm{~s}$ & $4.3 \mathrm{~s}$ & $5.9 \mathrm{~s}$ \\
\hline
\end{tabular}

It is worth noting that the setting time of the proposed finitetime algorithm depends on the parameter selection and the communication network structure. Once the size of the microgrid is increased, the network information may change so that the upper bounds on the setting time will change accordingly. However, the proposed algorithm solves the optimal resource management in a distributed and sparse approach, and hence, it is scalable for a large-scale network, which is verified in Case 5.4. Also, real islanded microgrids are usually smallscale due to the mission-critical application so that they usually have the limited inverters. The applicability of the proposed algorithm for an actual islanded system is tested in Case 5.3.

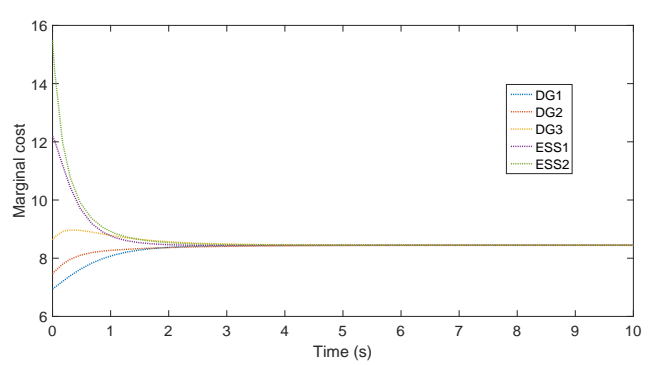

Fig. 5. The marginal cost update with the distributed gradient algorithm

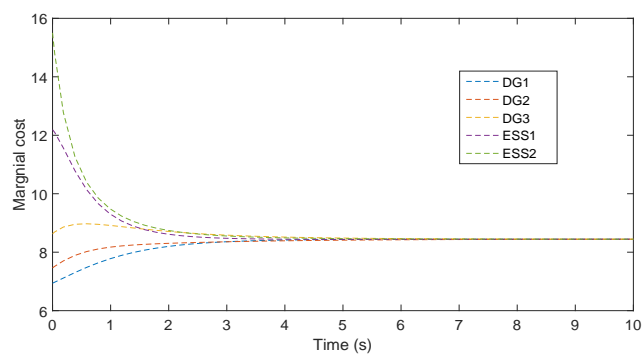

Fig. 6. The marginal cost update with the Laplacian-gradient dynamics

\section{B. Case 5.2}

As the results in Case 5.1 shown, the proposed algorithm is applicable for microgrids under the unpredictable change of the operation mode. Then, in this case, the proposed algorithm is applied to a microgrid under both islanded and grid-tied modes to verify the effectiveness of the proposed algorithm during different operation modes of the microgrid. The microgrid is supposed to be operated in islanded mode at $0 \mathrm{~s}$, and be connected to the grid at $5 \mathrm{~s}$. During the simulation study, we assume there exists a spanning tree in the communication network, which is used to ensure the convergence of the proposed algorithm. Fig. 7 and 8 give the updates of the marginal cost and actual output power during the microgrid operation. As Fig. 7 shown, the marginal cost of each dispatchable agent converges to the optimal value initially. After the microgrid is connected to the grid at $5 \mathrm{~s}$, the marginal cost converges to a new value because it is controlled to follow the reference set by the utility. The results show that both of them converge to the optimal value in a finite-time, and the setting time is bounded.

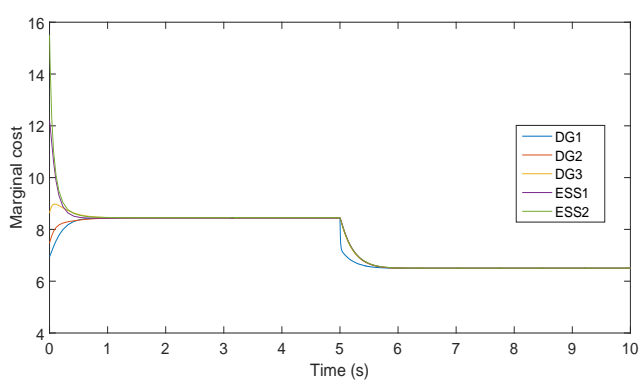

Fig. 7. The marginal cost update with the proposed finite-time algorithm 


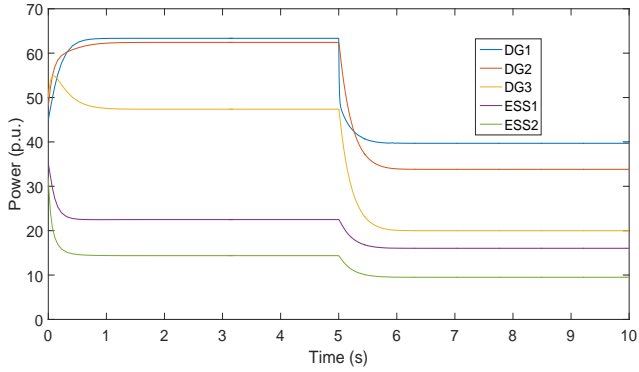

Fig. 8. The actual output power

\section{Case 5.3}

With the above results, the Case 5.3 is then adopted to demonstrate the potentiality of the practical application of the algorithm. In this case, an actual islanded system located in Inner Mongolia of China [35] is developed in MATLAB/Simulink to demonstrate the effectiveness of the proposed algorithm. The single line diagram of the islanded system is shown as Fig. 9, which consists of eight synchronous generators (SGs), two wind power plants and three aluminum smelting loads. The relevant configurations of each participant can be found in [36]. In this islanded system, the penetration of wind power generation is up to $30 \%$ so that the stability of the islanded system is very crucial. The stochastic wind power generations lead to a requirement for a fast convergence algorithm.

In this simulation study, the coefficients for SGs are analytically selected to proper practical conditions [36]. Three aluminum smelting loads are regarded as constant loads. Furthermore, the utilization of renewable resource should be as much as possible. As a result, the wind speed of two wind power plants is supposed to be appropriate that the maximum available power of these plants should be equal to their capacity, respectively.

By supposing the communication network is connected, Fig. 10 and 11 show that the marginal cost of each dispatchable agent converges to the optimal value firstly, and the actual power outputs converge to the optimal values accordingly. However, the islanded system may encounter the tripping problem of SGs. As a result, the algorithm is further adopted to verify the effectiveness of the algorithm when the SG is tripped. In the simulation, the SG4 is supposed to be suddenly tripped at $5 \mathrm{~s}$, and the rest of SGs then share the mismatch to maintain the supply-demand balance. Therefore, as shown in Fig. 10 and 11, the marginal cost converges to a new optimal value and actual power outputs changes to new optimal values in a faster convergence manner consequently after $5 \mathrm{~s}$. It is worth noting that the SG3 and SG5 firstly response to the tripping of SG4 as they are the adjacent of SG4.

Remark 5.1: The intermittency of the wind power generation would cause the supply-demand mismatch, which could be treated as a constant variation in a short time. In the Case 5.3, since the SG tripping may cause the load variation, by considering the SG tripping during the simulation, the results could verify the effectiveness of the algorithm for the load demand variation from other side. Meanwhile, the storage system can

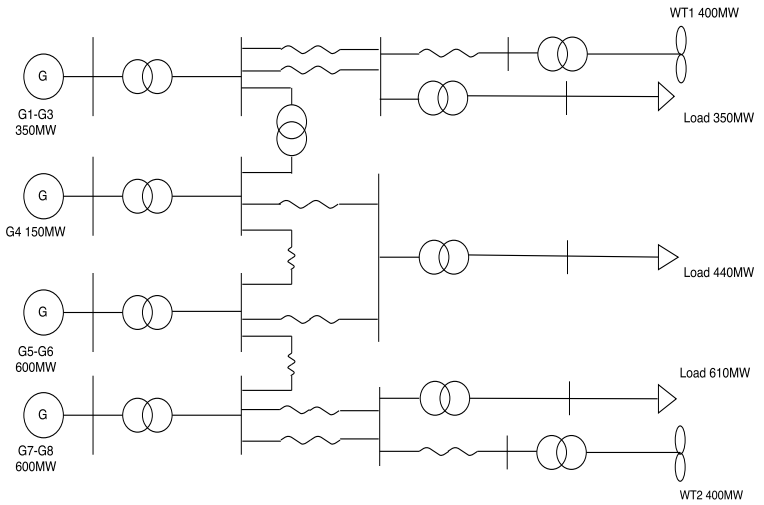

Fig. 9. An actual islanded system in China [35]

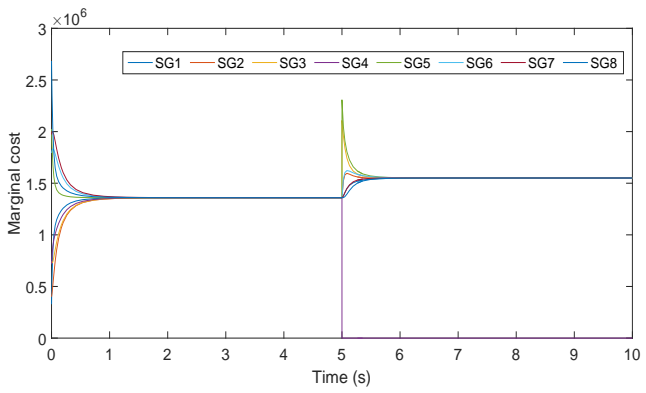

Fig. 10. The marginal cost update with the proposed finite-time algorithm for an actual islanded microgrid

be integrated into the system by deploying the formulated cost function and the proposed algorithm.

\section{Case 5.4}

In this case, the IEEE 162-bus system is adopted to further evaluate the performance of proposed strategy. The adopted system is modified with nine generators and eight ESSs and the initial condition is given by [32] with a total load demand of $18422 \mathrm{MW}$. The communication network is assumed weightbalanced and strongly connected.

Deploying the algorithm at 0s, the results in Fig. 12 and Fig. 13 show the proposed strategy can guarantee the marginal costs to converge to the optimal value while the allocated active powers to optimal values correspondingly. Thus the scalability of the proposed algorithm is demonstrated.

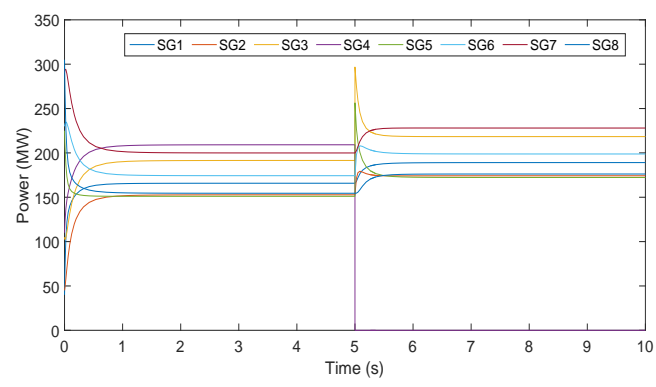

Fig. 11. The actual output power 


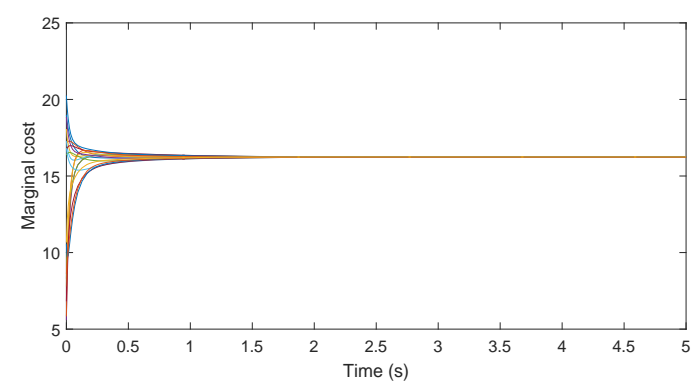

Fig. 12. The marginal cost update

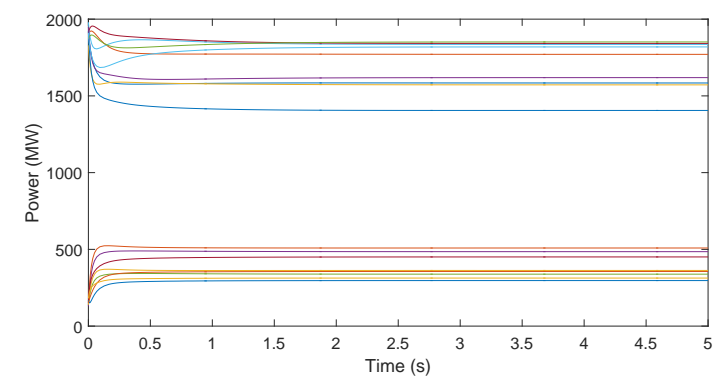

Fig. 13. The actual output power

\section{CONCLUSION}

In this paper, a MAS-based finite-time distributed optimization strategy is proposed for the optimal resource management in a microgrid under both islanded and grid-connected modes. The proposed strategy can minimize the generation cost of each participant while maintaining the supply-demand balance in a finite-time manner within a microgrid. Based on a MAS framework, the proposed strategy can work in a distributed manner without a central controller. The upper bounds of the convergence time are established by a rigorous proof, which guarantee the high speed convergence. The simulation results in the IEEE 14- and 162-bus system and an actual islanded system demonstrate that the potential practical ability of the proposed strategy for a real microgrid.

\section{REFERENCES}

[1] H. K. Nunna and S. Doolla, "Multiagent-based distributed-energyresource management for intelligent microgrids," IEEE Trans. Ind. Electron., vol. 60, no. 4, pp. 1678-1687, 2013.

[2] I. J. Balaguer, Q. Lei, S. Yang, U. Supatti, and F. Z. Peng, "Control for grid-connected and intentional islanding operations of distributed power generation," IEEE Trans. Ind. Electron., vol. 58, no. 1, pp. 147-157, 2011.

[3] Q. Shafiee, T. Dragič evi ć, P. Popovski, J. C. Vasquez, J. M. Guerrero et al., "Robust networked control scheme for distributed secondary control of islanded microgrids," IEEE Trans. Ind. Electron., vol. 61, no. 10, pp. 5363-5374, 2014.

[4] D. E. Olivares, A. Mehrizi-Sani, A. H. Etemadi, C. A. Cañizares, R. Iravani, M. Kazerani, A. H. Hajimiragha, O. Gomis-Bellmunt, M. Saeedifard, R. Palma-Behnke et al., "Trends in microgrid control," IEEE Trans. smart grid, vol. 5, no. 4, pp. 1905-1919, 2014.

[5] J. M. Guerrero, P. C. Loh, T.-L. Lee, and M. Chandorkar, "Advanced control architectures for intelligent microgrids-part ii: Power quality, energy storage, and ac/dc microgrids," IEEE Trans. Ind. Electron., vol. 60 , no. 4 , pp. 1263-1270, 2013.

[6] B. Wollenberg and A. Wood, "Power generation, operation and control," John Wiley\&Sons, Inc, pp. 264-327, 1996.
[7] A. Pantoja and N. Quijano, "A population dynamics approach for the dispatch of distributed generators," IEEE Trans. Ind. Electron., vol. 58, no. 10, pp. 4559-4567, 2011.

[8] A. Chaouachi, R. M. Kamel, R. Andoulsi, and K. Nagasaka, "Multiobjective intelligent energy management for a microgrid," IEEE Trans. Ind. Electron., vol. 60, no. 4, pp. 1688-1699, 2013.

[9] N. Jain, S. Singh, and S. Srivastava, "A generalized approach for DG planning and viability analysis under market scenario," IEEE Trans. Ind. Electron., vol. 60, no. 11, pp. 5075-5085, 2013.

[10] H. Xin, Z. Qu, J. Seuss, and A. Maknouninejad, "A self-organizing strategy for power flow control of photovoltaic generators in a distribution network," IEEE Trans. Power Syst., vol. 26, no. 3, pp. 1462-1473, 2011.

[11] S. Kar and G. Hug, "Distributed robust economic dispatch in power systems: A consensus+ innovations approach," in 2012 IEEE Power and Energy Society General Meeting. IEEE, 2012, pp. 1-8.

[12] S. Yang, S. Tan, and J.-X. Xu, "Consensus based approach for economic dispatch problem in a smart grid," IEEE Trans. Power Syst., vol. 28, no. 4, pp. 4416-4426, 2013.

[13] J.-H. Teng, S.-W. Luan, D.-J. Lee, and Y.-Q. Huang, "Optimal charging/discharging scheduling of battery storage systems for distribution systems interconnected with sizeable pv generation systems," IEEE Trans. Power Syst., vol. 28, no. 2, pp. 1425-1433, 2013.

[14] Z. Yang, R. Wu, J. Yang, K. Long, and P. You, "Economical operation of microgrid with various devices via distributed optimization," IEEE Trans. Smart Grid, vol. 7, no. 2, pp. 857-867, 2016.

[15] Y. Xu, W. Zhang, W. Liu, X. Wang, F. Ferrese, C. Zang, and H. Yu, "Distributed subgradient-based coordination of multiple renewable generators in a microgrid," IEEE Trans. Power Syst., vol. 29, no. 1, pp. 23-33, 2014.

[16] Y. F. Eddy, H. B. Gooi, and S. X. Chen, "Multi-agent system for distributed management of microgrids," IEEE Trans. Power Syst., vol. 30, no. 1 , pp. 24-34, 2015.

[17] Q. Li, C. Peng, M. Chen, F. Chen, W. Kang, J. M. Guerrero, and D. Abbott, "Networked and distributed control method with optimal power dispatch for islanded microgrids," IEEE Trans. Ind. Electron., vol. 64, no. 1, pp. 493-504, 2017.

[18] Z. Zhang and M.-Y. Chow, "Incremental cost consensus algorithm in a smart grid environment," in 2011 IEEE Power and Energy Society General Meeting. IEEE, 2011, pp. 1-6.

[19] Y. Xu and Z. Li, "Distributed optimal resource management based on the consensus algorithm in a microgrid," IEEE Trans. Ind. Electron., vol. 62, no. 4, pp. 2584-2592, 2015.

[20] M. Ç. Pinar and S. A. Zenios, "On smoothing exact penalty functions for convex constrained optimization," SIAM Journal on Optimization, vol. 4, no. 3, pp. 486-511, 1994.

[21] W. Zhang, W. Liu, X. Wang, L. Liu, and F. Ferrese, "Online optimal generation control based on constrained distributed gradient algorithm," IEEE Trans. Power Syst., vol. 30, no. 1, pp. 35-45, 2015.

[22] R. de Azevedo, M. H. Cintuglu, T. Ma, and O. Mohammed, "Multiagent based optimal microgrid control using fully distributed diffusion strategy," IEEE Trans. Smart Grid, 2016.

[23] C. Zhou, K. Qian, M. Allan, and W. Zhou, "Modeling of the cost of ev battery wear due to v2g application in power systems," IEEE Trans. Energy Convers., vol. 26, no. 4, pp. 1041-1050, 2011.

[24] Y. Zhang and M.-Y. Chow, "Microgrid cooperative distributed energy scheduling (codes) considering battery degradation cost," in Industrial Electronics (ISIE), 2016 IEEE 25th International Symposium on. IEEE, 2016, pp. $720-725$.

[25] S. Boyd and L. Vandenberghe, Convex optimization. Cambridge university press, 2004.

[26] S. S. Kia, "Distributed optimal resource allocation over networked systems and use of an e-exact penalty function," IFAC-PapersOnLine, vol. 49, no. 4, pp. 13-18, 2016.

[27] L. Wang and F. Xiao, "Finite-time consensus problems for networks of dynamic agents," IEEE Trans. Autom. Control, vol. 55, no. 4, pp. 950-955, 2010.

[28] Z. Zuo and L. Tie, "A new class of finite-time nonlinear consensus protocols for multi-agent systems," International Journal of Control, vol. 87, no. 2, pp. 363-370, 2014.

[29] Z. Ding, Nonlinear and adaptive control systems. IET, 2013, vol. 84.

[30] S. Zuo, A. Davoudi, Y. Song, and F. L. Lewis, "Distributed finitetime voltage and frequency restoration in islanded ac microgrids," IEEE Trans. Ind. Electron., vol. 63, no. 10, pp. 5988-5997, 2016.

[31] Y. Xu, W. Zhang, G. Hug, S. Kar, and Z. Li, "Cooperative control of distributed energy storage systems in a microgrid," IEEE Trans. Smart Grid, vol. 6, no. 1, pp. 238-248, 2015.

[32] P. S. T. C. Archive:,"162-bus, 17 generator dynamic test case," http: //www.ee.washington.edu/research/pstca/dyn17/pg_tcadd17.htm. 
[33] Z. Fu, X. He, T. Huang, and H. Abu-Rub, "A distributed continuous time consensus algorithm for maximize social welfare in micro grid," Journal of the Franklin Institute, vol. 353, no. 15, pp. 3966-3984, 2016.

[34] A. Cherukuri and J. Cortés, "Distributed generator coordination for initialization and anytime optimization in economic dispatch," IEEE Trans. Control Netw. Syst., vol. 2, no. 3, pp. 226-237, 2015.

[35] H. Jiang, J. Lin, Y. Song, W. Gao, Y. Xu, B. Shu, X. Li, and J. Dong,
"Demand side frequency control scheme in an isolated wind power system for industrial aluminum smelting production," IEEE Trans. Power Syst., vol. 29, no. 2, pp. 844-853, 2014.

[36] Z. Wang, W. Wu, and B. Zhang, "A fully distributed power dispatch method for fast frequency recovery and minimal generation cost in autonomous microgrids," IEEE Trans. Smart Grid, vol. 7, no. 1, pp. 19-31, 2016. 